\title{
Des possibilités d'emploi du sperme de taureau transporté de France pour l'insémination artificielle dans les territoires d'outre-mer
}

\author{
par \\ E. LETARD, P. SZUMOWSKI et $M$. PAREZ (France) \\ M. PAGOT, Z. DERBAL, J. BALLIS et J. SOUQUET (A.O.F.)
}

\section{I. - INTRODUCTION*}

Il $\mathrm{y}$ a $=n$ Afrique Occidentalo Françaiac (A.O.F.) cnviron 6.000 .000 ij Ejvins, dont 4.000 .000 de zébus et 2.000 .000 de taurins; lé:= emélicration fut tentée par croisements, dès 1927, par le Servize de l'Élevage et des Industries Animales qui importa au Souda: $=$ Es taureaux charrollais et normands et un peu plus tard en Guinee des taureaux tarentais. En 1938, le Service de l'Élevage $\dot{s} \in$ iOffice du Niger importait à son tour des taureaux charollais $\equiv:$ :.:ntbéliard.

Les pertes parmi les reproducteurs pur sang furent, dans toutes ces $\mathrm{x}_{\mathrm{F}}$ èr:ences, très sévères; aussi était-il normal que l'on pensât ミ̇ útiliser l'insémination artificielle pour obtenir économiquement des métis dans les territoires outre-mer.

Des essa:s a insémination artificielle dans l'espèce bovine avec du sperme :=ansporté à longue distance ont été tentés dans quelques pays étrangers. La fécondation a été réussie avec du sperme expédié par avion dans quelques cas. Nous rappelons ci les performances les plus significatives :

$1^{0}$ Transport de sperme de taureau de Everittstown (U.S.A.) a Sidney (Australie); distance de 26.000 kilomètres, durée du transport cinq jours; inséminations faites avec succès.

20 Transport du sperme de taureau de Beltsville (U.S.A.) à Piran (Argentine) et fécondation réussie avec ce sperme, sept jours après la recclte.

$3^{\circ}$ Trente-sinq veaux obtenus, en 1948, à la suite d'un expédition de sperme des États-Unis en Italie.

Ces essais sporadiques furent faits à titre expérimental, sans tenir compt $\triangleq$ jes frais de transport et pour l'insémination des vaches dans jes régions plus ou moins tempérees.

Mais à côte de ces réussites, d'autres essais furent beaucoup moins enccurzzeants, et des échecs nombreux ont été enregistrés sans que la zäuse ait pu en être reconnue.

Nous présentons ici les résultats de nos recherches sur les possibilités pratiques d'emploi de l'insémination artıficielle, en zone tropicale, à l'aide d'expéditions à grandes distances faites de façon réguliere. Le sperme a été récolté en France et transporté en A.O.F. periodiquement, au cours de trois années. La distance etait à vol d'oiseau de 4.335 kilometres pour Bamako et 4.030 kilomètres pour Niamey.

Notre but était le suivant :

10 Prouver l'efficacité de la méthode du conditionnement pour l'envoi de sperme à longue distance,

* N. B. Les expériences relatées dans cet article n'impliquent en rien que le croisement constitue la methode à choisir pour l'amélioration du cheptel bovin d'A. O.F. telle que l'avait mise au point auparavant le Laboratoire d'Alfort (1-1949).

$2^{\circ}$ Déterminer les possibilités d'expédition régulière du sperme de taureau en A.O.F. dans des conditions économiques, c'est-à-dire avec des frais de transport aussi réduits que possible, à l'aide d'un emballage très léger.

Les essais, commencés en 1949, furent faits grâce à l'étroite collaboration du Laboratoire de zootechnie de l'Institut national de la recherche agronomique rattaché à l'École nationale vétérinaire d'Alfort, du Centre de génétique animale de l'Office de la recherche scientifique d'outre-mer, du Service de l'elevage et des industries animales de l'A.O.F., du Centre d'insémination de la Coopérative d'élevage de Magny-en-Vexin, et de l'Office du Niger.

\section{II. - TECHNIQUE DE L'ENVOI DE SPERME}

Quatre séries d'envois furent faites. Dans les trois premières, la semence était expédiée de Paris par le Laboratoire précité d'Alfort, la dernière série fut expédiée de Magny-en-Vexin, le transit à Paris vers un aérodrome étant assuré, dans ce dernier cas, par un transitaire privé.

Les envois ont été faits sur Bamako (Soudan) et Filingué (Niger), localités où le Service de l'élevage possède des fermes expérimentales et sur Ségou, via Bamako, où l'Office du Niger possède une station expérimentale.

Récolte, examen et dilution des échantillons. Plusieurs taureaux furent utilisés : 1 schwitz, I normand, I montbéliard, 1 tarentais, 1 charoliais et 1 jerseyais, tous éprouvés à l'égard de la brucellose. La récolte était faite avec le vagin artificiel de modèle anglais. Immédiatement après la récolte, la motilité, la densité, le $\mathrm{pH}$, le nombre de spermatozoïdes par millimètre cube (concentration) étaient déterminés, le test de la réductase était pratiqué. 
TABLEAU I

Qualité du sperme au départ d'après l'examen du Laboratoire d'Alfort

\begin{tabular}{|c|c|c|c|c|c|c|c|c|c|c|c|c|}
\hline \multirow{3}{*}{$\begin{array}{c}\text { CARACTĖRES } \\
\text { du } \\
\text { sperme }\end{array}$} & \multicolumn{12}{|c|}{ TAUREAUX } \\
\hline & \multicolumn{2}{|c|}{ Schwitz } & \multicolumn{2}{|c|}{ Montbéliard } & \multicolumn{2}{|c|}{ Normand } & \multicolumn{2}{|c|}{ Tarentais } & \multicolumn{2}{|c|}{ Charollais } & \multicolumn{2}{|c|}{$\begin{array}{l}\text { Moyenne des } \\
\text { cinq taureaux }\end{array}$} \\
\hline & Moyenue & Yariatiogs & Movenne & Yariations & Joyenge & Tariations & Ilogerne & Pariations & Moyento & Yarintions & Moyenne & Forialions \\
\hline Volume cc. & 4,4 & $2,7-6,2$ & 5,5 & $2,5-10$ & 4,9 & $1,6-7,5$ & 3,2 & $2,5-4,0$ & 4,8 & $2,5-6,0$ & 4.7 & $1,6-10$ \\
\hline Motilité $\% \ldots .$. & 77 & $.70-85$ & 77 & $65-85$ & 81 & $60-90$ & 81 & $80-85$ & 87 & $85-90$ & 81 & $60-90$ \\
\hline $\begin{array}{l}\text { Concentration en } \\
\text { millions par } \mathrm{mm}^{3}\end{array}$ & 1,74 & $\mathbf{1}, 26-2,2$ & 1,20 & $0,37-2,20$ & 1,48 & $0,73-1,98$ & 1,41 & $1,13-1,91$ & 2,19 & $1,28-2,78$ & 1,60 & $0,37-2,78$ \\
\hline $\begin{array}{l}\text { Test de la réduc- } \\
\text { tase. }\end{array}$ & & & & & & & & & & & & \\
\hline $\begin{array}{l}\text { 10 Sperme non } \\
\text { dilue (en minutes } \\
\text { et secondes) ... }\end{array}$ & $3^{\prime} 40^{\prime \prime}$ & $11^{\prime} 30^{\prime \prime}-6$ & $5^{\prime} 40^{\prime \prime}$ & $2^{\prime} 30^{\prime \prime}-11^{\prime}$ & $3^{\prime} \pm 0^{\prime \prime}$ & $2^{\prime}-7^{\prime} 30^{\prime \prime}$ & $348^{\prime \prime}$ & $2^{\prime}-4^{\prime} 30^{\prime \prime}$ & 2'18" & $1^{\prime} 45^{\prime \prime}-33^{\prime}$ & $357^{\prime \prime}$ & $1^{\prime} 30-11^{\prime}$ \\
\hline $\begin{array}{l}2^{\circ} \text { Sperme dilué } \\
(1: 5) \ldots \ldots \ldots \ldots\end{array}$ & $14^{\prime} \pm 0^{\prime \prime}$ & $7^{\prime} 30^{\prime \prime}-31^{\prime}$ & $17 ' 50$ ' & $6^{\prime}-41^{\prime}$ & $8^{\prime} 50^{\prime \prime}$ & $6^{\prime}-16^{\prime}$ & $10^{\prime}$ & $8^{\prime}-12^{\prime}$ & $5^{\prime} 6^{\prime \prime}$ & $5^{\prime}-5^{\prime} 30^{\prime \prime}$ & $11^{\prime} 9^{\prime}$ & $\bar{s}^{\prime}-41^{\prime}$ \\
\hline $\mathrm{pH} \ldots \ldots \ldots$ & 6,3 & $6,2-6,5$ & 6,3 & $6, \mathbf{1 - 6 , 6}$ & 6,3 & $6,1 \cdot 6,4$ & 6,3 & $6,3 \cdot 6,4$ & 6,3 & $6,1-6,4$ & 6,3 & $6,1-6,6$ \\
\hline
\end{tabular}

TABLEÃU II

Évolution des qualités du sperme dilué, après le transport et la conservation (cing premiers envois)

\begin{tabular}{|c|c|c|c|c|c|c|}
\hline \multirow[b]{2}{*}{$\begin{array}{l}\text { INOMBRE D'HEURES } \\
\text { après la récolte }\end{array}$} & \multicolumn{2}{|c|}{ TAUREAU SCHWITZ } & \multicolumn{2}{|c|}{ TEUREAU MONTBÉLIARD } & \multicolumn{2}{|c|}{ TAUREAU NORMAND } \\
\hline & $\begin{array}{c}\text { Variations } \\
\text { de Ia motilité \% }\end{array}$ & $\begin{array}{c}\text { Variations } \\
\text { du temps } \\
\text { de la réductase. } \\
\text { Minute Seconde. }\end{array}$ & $\begin{array}{c}\text { Variations } \\
\text { de la motilité } \%\end{array}$ & \begin{tabular}{|c|} 
Variations \\
du temps \\
de la. réductase \\
Minute Seconde.
\end{tabular} & 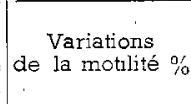 & $\begin{array}{l}\text { Variations } \\
\text { du temps } \\
\text { de la réductase } \\
\text { Minute Seconde }\end{array}$ \\
\hline $0 \ldots \ldots \ldots \ldots$ & $70-85$ & $5^{\prime}-\ldots 12^{\prime}$ & $65-85$ & $8^{\prime}-30^{\prime}$ & $75-80$ & $7^{\prime}-12^{\prime} 30^{\prime \prime}$ \\
\hline $42-64 \ldots \ldots \ldots \ldots$ & $70-75$ & $13^{\prime}-13^{\prime} 10^{\prime \prime}$ & $65-85$ & $\left(9^{\prime}\right)$ & $\gg$ & $(11)$ \\
\hline $88-112 \ldots \ldots$ & $60-65$ & $16^{\prime} .18^{\prime}$ & $60-70$ & $\left(10^{\prime}\right)$ & $60-75$ & $9^{\prime}-14^{\prime}$ \\
\hline $134-158 \ldots \ldots$ & $\gg$ & $»$ & $50-60$ & $\gg$ & $(50)$ & $»$ \\
\hline $172-188$. & $\gg$ & $\gg$ & $30-40$ & 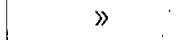 & (30) & $\gg$ \\
\hline $198-232 \ldots \ldots$ & $\gg$ & $»$ & $20-50$ & $20^{\prime}-60^{\prime}$ & $(20)$ & $\gg$ \\
\hline $12-13$ jours.... & $\begin{array}{l}\text { quelques } \\
\text { vivants }\end{array}$ & $\gg$ & $\begin{array}{l}\text { quelques } \\
\text { vivants }\end{array}$ & ${ }^{\prime \prime}$ & $\begin{array}{l}\text { quelques } \\
\text { vivants }\end{array}$ & $: \quad \gg$ \\
\hline 22 jours ......... & $\gg$ & $\gg$ & $\begin{array}{l}\text { quelques } \\
\text { vivants }\end{array}$ & , & $》$ & $\gg$ \\
\hline
\end{tabular}

Le sperme était dilué, suivant les échantillons, dans 4 à 9 parties de dilueur citrate jaune d'œuf, addiiionné de 3 milligrammes de sulfaimide ( $1.162 \mathrm{~F}$ ) par centimètre cube de ce dilueur.

La dilution était mise dans des tubes de 15 à $20 \mathrm{cc}$. puis était recouverte d'huile de paraffine de telle façon que le bouchon du tube arrive au contact de l'huile quand' on l'introduisait.

Le remplissage bien complet et le bouchage hermétique (sans bulles d'air) de ces tubes permettaient d'éviter l'agitation du, sperme au cours du transport. 


\section{PLANCHE I}

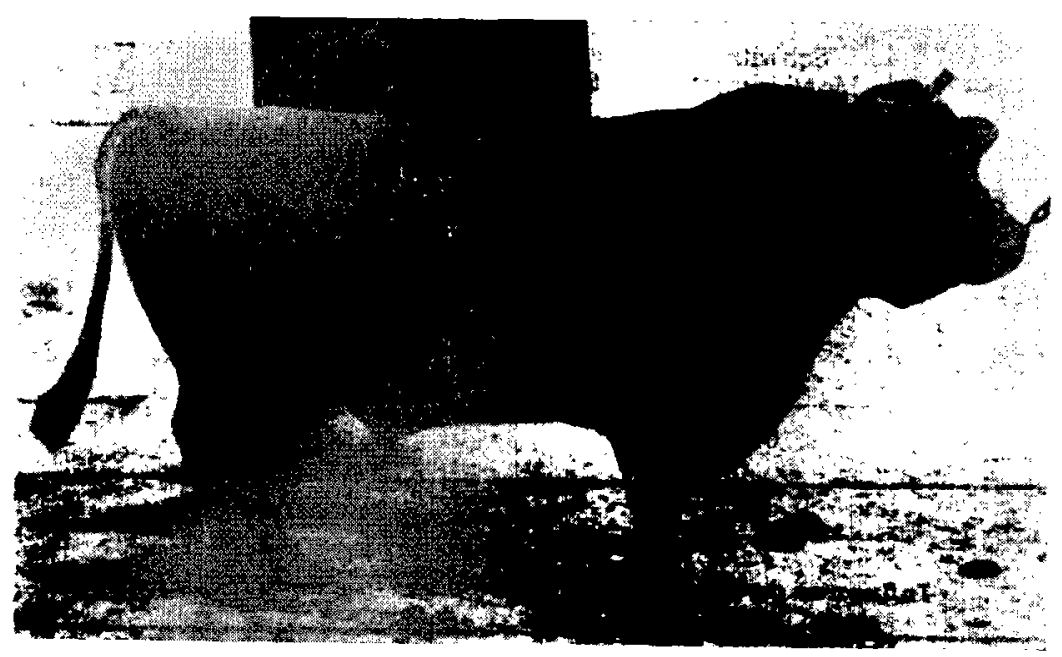

Un des taureaux utilistes. Jersiais du centre de Magny-en-Vexin.

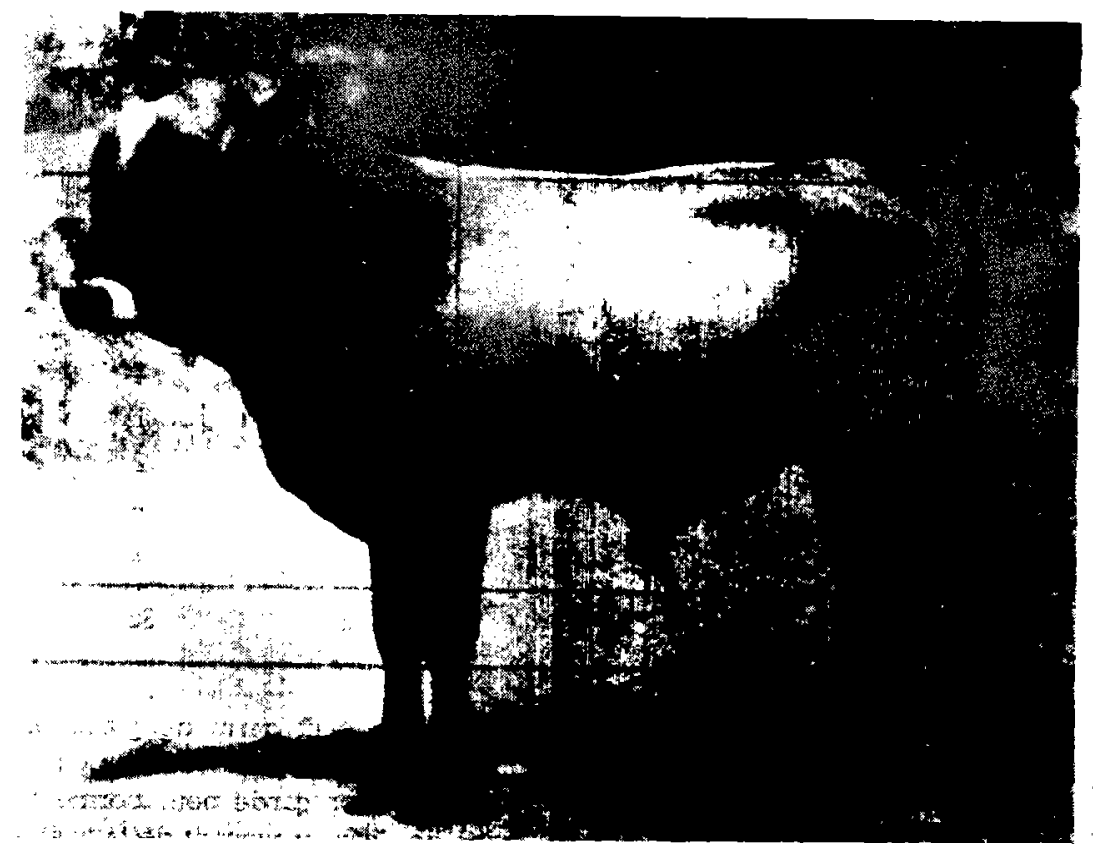

Premier produit né à Sotuba. Veau demi-sang Schwitz-n'Dama, né le 15/5/1950, photographié à 10 mois. 
TABLEAUU III

\begin{tabular}{|c|c|c|c|c|c|c|c|c|}
\hline 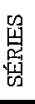 & $\begin{array}{l}\text { DATES D'ENVOIS } \\
\text { du sperme }\end{array}$ & $\begin{array}{l}\text { TAUREAUX } \\
\text { utilisés }\end{array}$ & $\begin{array}{l}\text { NOMBRE } \\
\text { d'envois }\end{array}$ & $\begin{array}{c}\text { NOMBRE } \\
\text { de vaches } \\
\text { inséminées } \\
\text { (total) }\end{array}$ & $\begin{array}{c}\text { NOMBRE } \\
\text { de vaches } \\
\text { inséminées } \\
\text { avar.t } 96 . \mathrm{h} . \\
\end{array}$ & $\begin{array}{l}\text { NOMBRE } \\
\text { de vaches } \\
\text { fécondées }\end{array}$ & $\begin{array}{c}\% \\
\text { des vaches } \\
\text { fécondées } \\
\text { (total) }\end{array}$ & $\begin{array}{c}\% \\
\text { des vaches } \\
\text { fécondées } \\
\text { avant } 96 \mathrm{~h} .\end{array}$ \\
\hline 1 & dis $19-8$ alı $16-10-49 \ldots$ & $\begin{array}{c}\text { Schwitz } \\
\text { Montbéliard } \\
\text { Normand }\end{array}$ & 8 & 18 & 12 & 1 & 5,6 & 8,5 \\
\hline 2 & du $8-4$ au $16-5-50 \ldots$. & $\begin{array}{c}\text { 'Montbéliard } \\
\text { Normand } \\
\text { Tarentais }\end{array}$ & 3 & 11 & 9 & 5 & 45,5 & 56,0 \\
\hline 3 & du $18-12-50$ au $11-1-51 .$. & $\begin{array}{c}\text { Montbéliard } \\
\text { Charollais }\end{array}$ & 4 & 20 & 9 & 6 & 30,0 & 67,0 \\
\hline \multirow[t]{2}{*}{4} & du $18-6$ au $2-8-51 \ldots \ldots$ & Jersey & 6 & 8 & 8. & 3 & 37,5 & 37,5 \\
\hline & & 6 & 21 & 57 & 38 & 15 & 29,7 & 42,3 \\
\hline
\end{tabular}

TABLEAU IV.

Influence de l'âge de la semence sux les fécondations.

\begin{tabular}{|c|c|c|c|c|c|}
\hline \multirow{2}{*}{$\begin{array}{c}\text { A.GE DU SPERME } \\
\text { (heures) }\end{array}$} & \multicolumn{2}{|c|}{ INSÉMINATIONS FÉCONDES } & \multicolumn{3}{|c|}{ INSEMINA TIONS INFÉCONDES } \\
\hline & $\begin{array}{l}\text { Première } \\
\text { insémination }\end{array}$ & $\begin{array}{l}\text { Deuxième } \\
\text { insémination }\end{array}$ & $\begin{array}{l}\text { Premiere } \\
\text { insémination }\end{array}$ & $\begin{array}{l}\text { Deuxième } \\
\text { insémination }\end{array}$ & $\begin{array}{c}\text { Troisième } \\
\text { insémination }\end{array}$ \\
\hline $10-29$ & $\gg$ & $\gg$ & 2 & $\gg$ & $\gg$ \\
\hline $30-49$ & 3 & 3 & 4 & 2 & $\gg$ \\
\hline $50-69$ & 8 & 8 & 11 & 6 & $»$ \\
\hline $70-89$ & $》$ & $\gg$ & 7 & 5 & $\gg$ \\
\hline $90-109$ & 3 & 2 & 10 & 5 & 1 \\
\hline $\begin{array}{l}\text { Total jusqu'au } \\
\text { quatrième jour. .... }\end{array}$ & 14 & 13 & 34 & 18 & 1 \\
\hline $110-129$ & 1 & 1 & 2 & 5 & $\gg$ \\
\hline $130-149$ & $»$ & $»$ & 4 & 4 & 1 \\
\hline $150-169$ & $\gg$ & $»$ & 1 & 1 & $»$ \\
\hline $170-189$ & $\gg$ & $»$ & 2 & 2 & $\gg$ \\
\hline $190-209$ & $\gg$ & $\gg$ & 6 & 4 & $\gg$ \\
\hline plus de 210 & $\gg$ & $\gg$ & 3 & ". 1 . & $»$ \\
\hline TOTAL..... & 15 & 14 & 52 & 35 & 2 \\
\hline
\end{tabular}

Aux deux premiers envois était joint un échantillon de sperme non dilué.

Emballage. - Les tubes de sperme dilué, entourés de coton cardé sec, étaient enveloppés dans des vessies en caoutchouc, cela afin d'éviter leur contact direct avec la glace. Puis, ils étaient mis dans des bouteilles Thermos d'un litre et demi. Le fond de la bouteille était garni de glace brisée en menus morceaux, mélangée avec un peu de sel, et recouverte de coton cardé pour permettre une recongélation de l'eau provenant de la fusion de la glace se trouvant au-dessus. L'espace restant libre dans la bouteille était comblé par des morceaux de glace entourés de coton.

Les bouteilles Thermos furent, au début, mises 


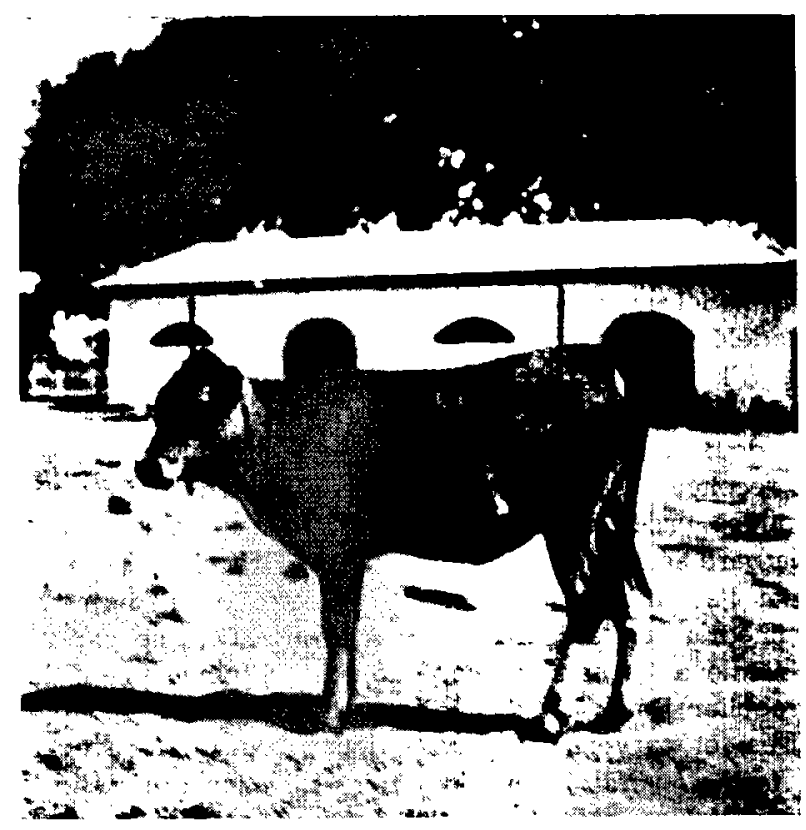

Génisse pur sang n'Dama, née le 15/5/50, photographiée à 10 mois.

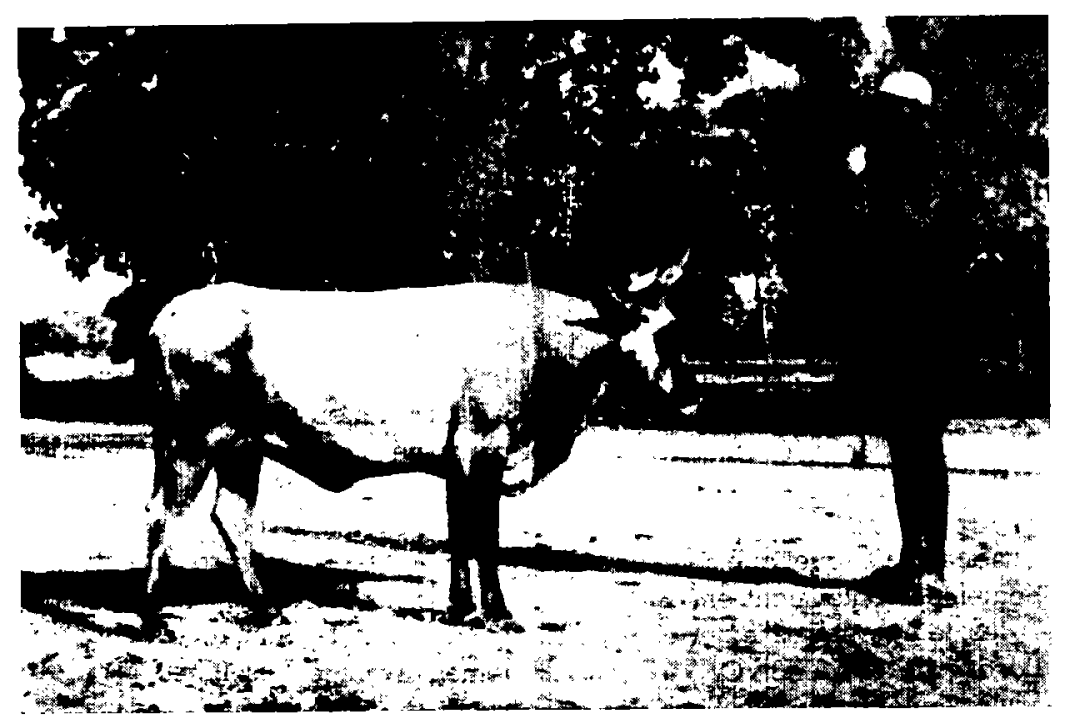

Mère du métis demi-sang Schwitz-n'Dama. 
dans des caissettes de bois blanc $(40 \times 20 \times 18 \mathrm{~cm}$.) dans lesquelles elles étaient calées par de la laine de verre et de la sciure de liège qui servaient d'isolants ; puis, les boîtes en bois furent remplacées d'abord par des cylindres de carton épais (diamètre $18 \mathrm{~cm}$., hauteur $45 \mathrm{~cm}$., épaisseur $3 \mathrm{~mm}$.) puis par de simples boîtes de carton ondulé $(40 \times 14 \times 14 \mathrm{~cm}$.) La laine de verre fut remplacée par le kapok, beaucoup plus léger.

Les caissettes pesaient, remplies, 4 kilos; les tonnelets de carton $3 \mathrm{~kg} 500$ et les boîtes en carton

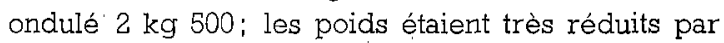
comparaison avec ceux des modòles antćrieurement conçus, par exemple du modèle américain dont nous disposions, et pesant vide, $8 \mathrm{~kg} 350$.

Horaires. - Pour les deux premières séries d'envois la semence était récoltée à Alfort, le vendredi soir vers 18 heures, diluée, mise en emballages isothermes et déposée à la gare aérienne d'Orly le scir même; elle partait par l'avion régulier d'Air France Paris-Dakar, le samedi matin à 8 heures, arrivait à Dakar le soir même à 23 heures, était réexpédiée par l'avion régulier Dakar-Bamako le dimanche matin à 6 heures, et parvenait à cette dernière ville le jour même, vers $10 \mathrm{~h}, 30$, soit environ quarante-deux heures après la récolte.

En 1950 et 1951, la création d'une ligne ParisCasablanca-Ramako-Abidjan, par Air France et l'Union aérienne de Transport, réduisit les délais de transport pour les dernières séries. Ainsi en juillet 1951, la semence récoltée à Magny-en-Vexin à 7 heures gagnait Paris par le train régulier vers midi, était transportée par un transitaire de la gare des Batignolles à Orly ou au Bourget d'où elle partait parr un courrier régulier à $17 \mathrm{~h} .30$ : elle arrivait à Bamako le lendemain matin vers: 7 h. 30 , soit vingtqualre heures après sa récolte:

En 1949-1950, la fréquence des courriers avion ne permettait qu'un envoi par semaine. En 1951, Bamako fut approvisionné deux fois par semaine, en juillet-août, les mardi et vendredi.

Pour Ségou, la semence reçue par l'avion ParisBamako était acheminée, après recharge en glace des bouteilles Thermos, par camion ou voiture légère.

Résultats. - A chaque fois et quiel que soit l'emballage utilisé, les bouteilles contenaient encore de la glace à l'arrivée, les températures extérieures en A.O.F. étant de l'ordre de $35^{\circ}$ à $43^{\circ} \mathrm{C}$.

La semence parvint toujours en excellent état de conservation.

Nous avons tenu à rester dans les conditions normales d'emploi; dès réception, lo contenu des bouteilles était vérifié, la charge de glace était complétée, les bouteilles étaient mises dans une armoire frigorifique à $+70 \mathrm{C}$.

Avec les échantillons de la première série d'envois, nous avons étudié l'évolution des qualités du sperme en fonction du temps, après des transports variant de quarante-deux à: soixante heures.

Les tableaux I et II indiquent les qualités du sperme à la récolte et après transport et conservation.

Comme le montrè le tableau I, les qualités du sperme des 5 taureaux utilisés pour l'insér ination des vaches en A.O.F. ont été, en général, d'une valeur supérieure à la moyonne. Lc ta'rrcau charollais, en particulier, a donné un très bon sperme.

D'après l'examen de quelques échantillons de sperme non dilué envoyé en A.O.F., on peut penser que ce sperme, 'ayant subi le transport aérien, 'ne resterait pas vivant au-delà de soixante-quatre heures après la récolte.

La motilité du sperme dilué au moment de l'arrivée en A.O.F. ne paraît nullement affectée. Mais quatre-vingt-huit à cent douze heures (quatre jours environ) après la récolte, elle tombe à la limite' inférieure compatible avec l'utilisation courante pour l'insémination artificielle.

Ainsi l'insémination avec le sperme dilué au jaune d'œuf citrate conservé au-delà de quatre jours, est-elle peu rationnelle, les chances de fécondation étant dans ce cas, en général, très réduites.

La survie du sperme dilué, après le transport en A.O.F., peuut néanmoins se prolonger jusqu'à douze, à treize jours (taureaux schwitz et normand) et même jusqu'à vingt-deux jours (taureau montbéliard) puisqu'on a encore trouvé au bout de ce temps, quelques spermatozoïdes vivants.

L'évolution de la motilité avec le temps ne semble guère influencée par le transport aérien, puisque la motilite du sperme de 3 taureaux à cent cinquantehuit heures de conservation au Laboratoire d'Alfort a été évaluée à $30-50 \%$.

L'épreuve au bleu de methylène, faite en A.O.F. à des températures d'étude variables (entre $33^{\circ}$ et $40^{\circ} \mathrm{C}$.), ne peut fournir des données comparables à celles qui ont été obtenues à Alfort (à $40^{\circ} \mathrm{C}$.). Elle confirme cependant que le sperme au-delà de quatre jours (88-112 heures) de conservation ne peut être utilisé pour l'insémination, le temps de décoloration ayant presque doublé.

Dans les séries suivantes d'envois, on se borna à déterminer la motilité des échantillons immédiatement avant les inséminations ; l'évolution fut comparable à celle constatée dans la première sárie d'envois. Quand le sperme expédié fut de bonne qualité, la conservation dans les conditions exposées précódemment fut excellente. 
PLANCHE III

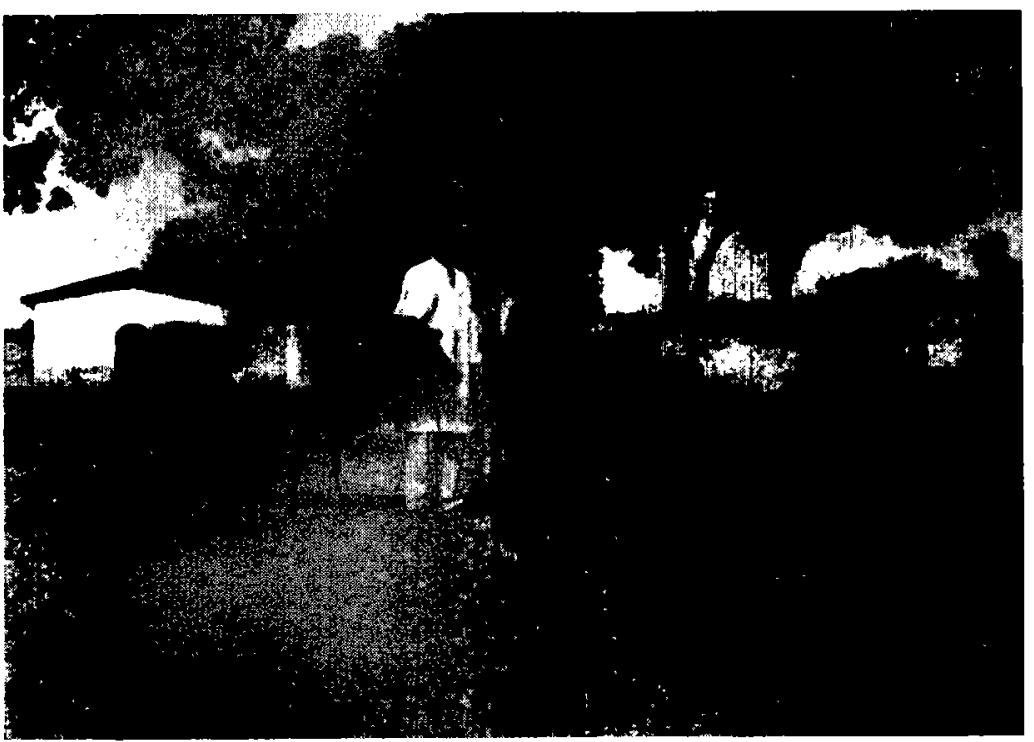

Mère du demi-sang Schwitz-n'Dama, photographiée avec son produit ágé de 18 mois.

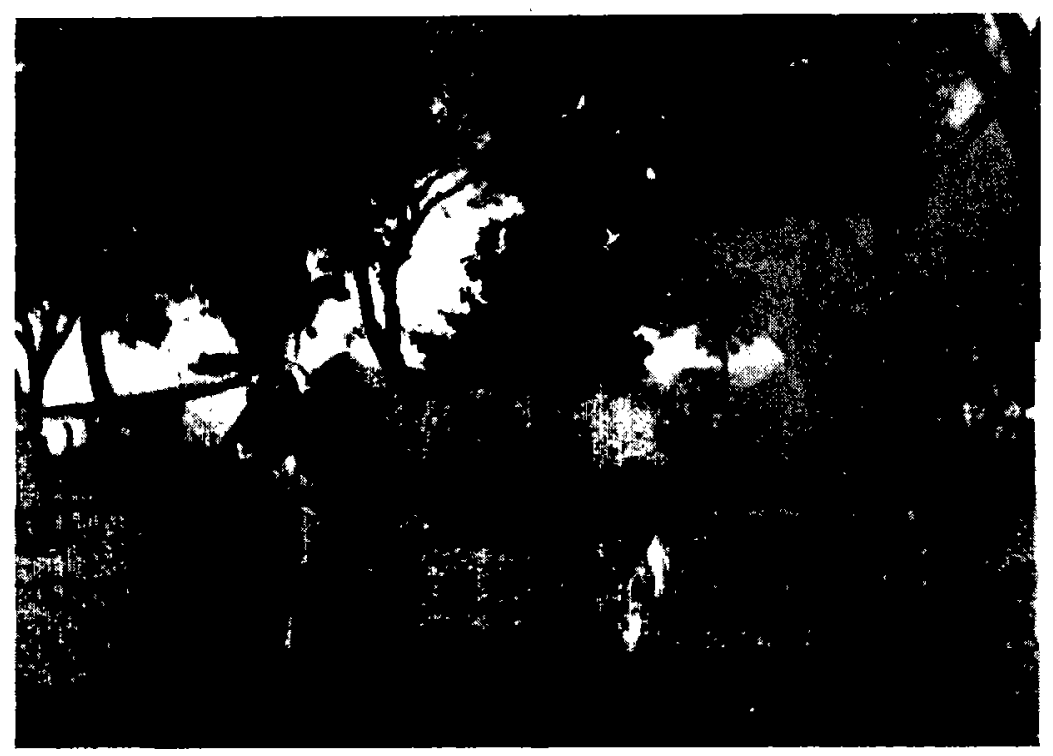

Mère de la génisse demi-sang Tarentais-n'Dama née le $23: 12 ; 50$. 


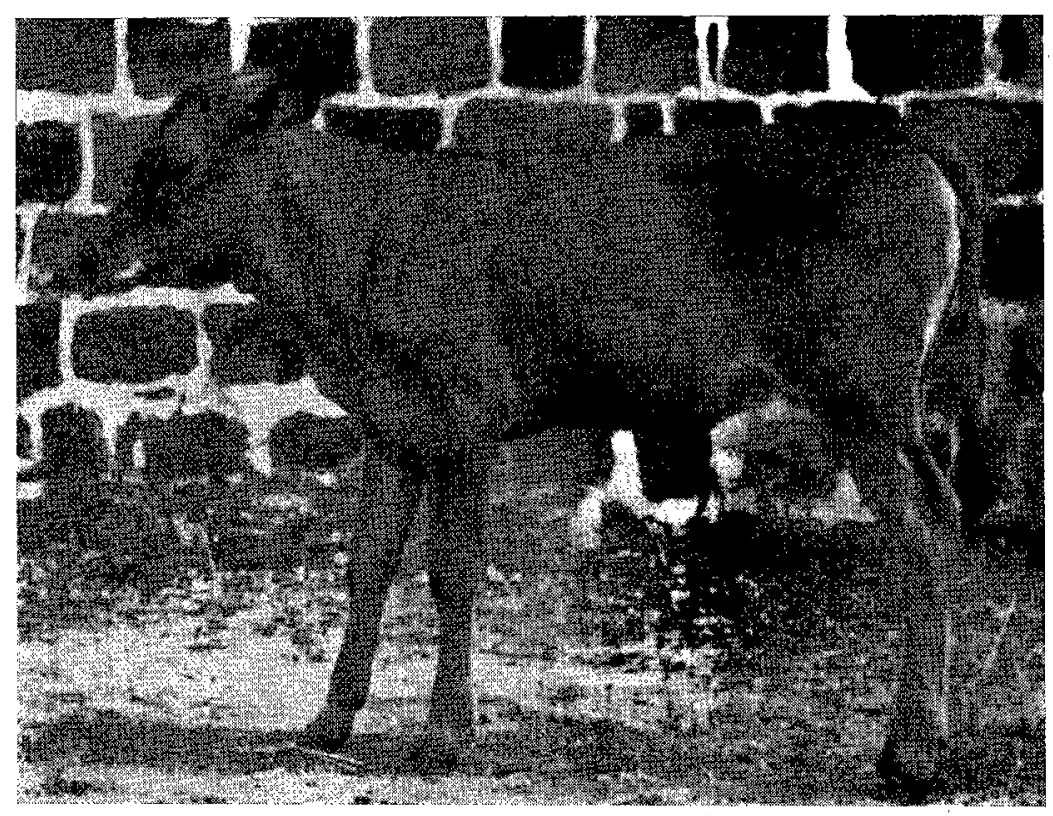

Génisse demi-sang Tarentais-n'Dama, néle 23/12/50, photog raphiée à 4 mois.

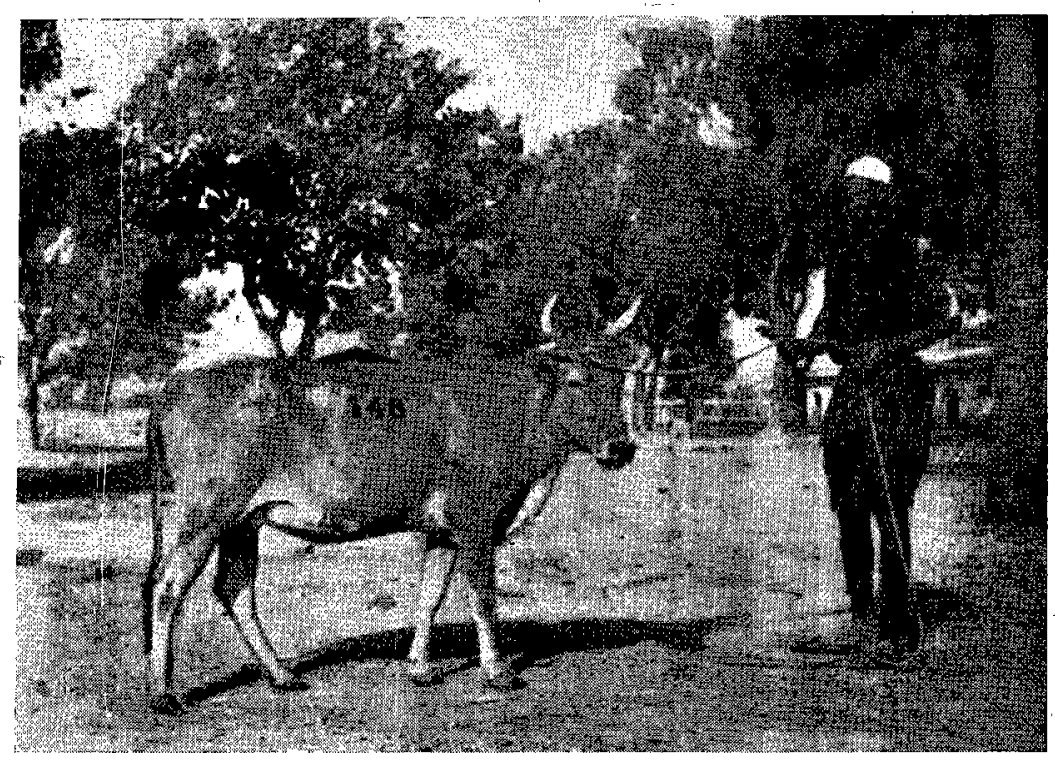

Mère du demi-sang Montbéliard-n'Dama né le 24/8/1951. 


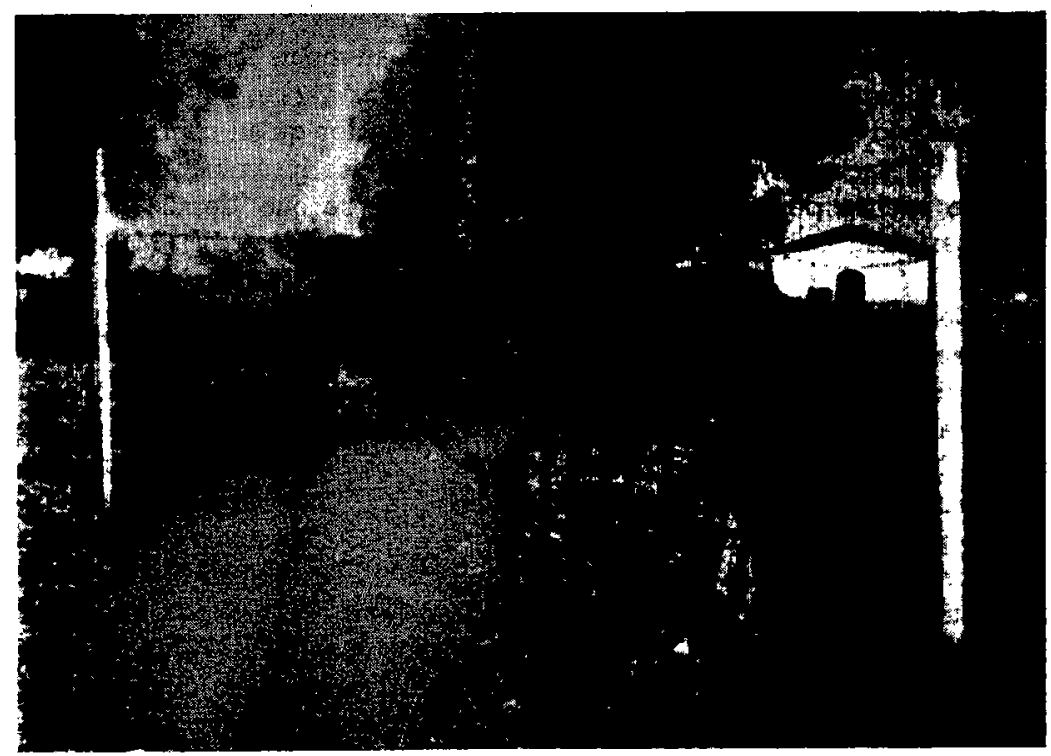

Demi-sang Montbéliard-n'Dama, né le 24 8'7951, photographié à 5 mois.

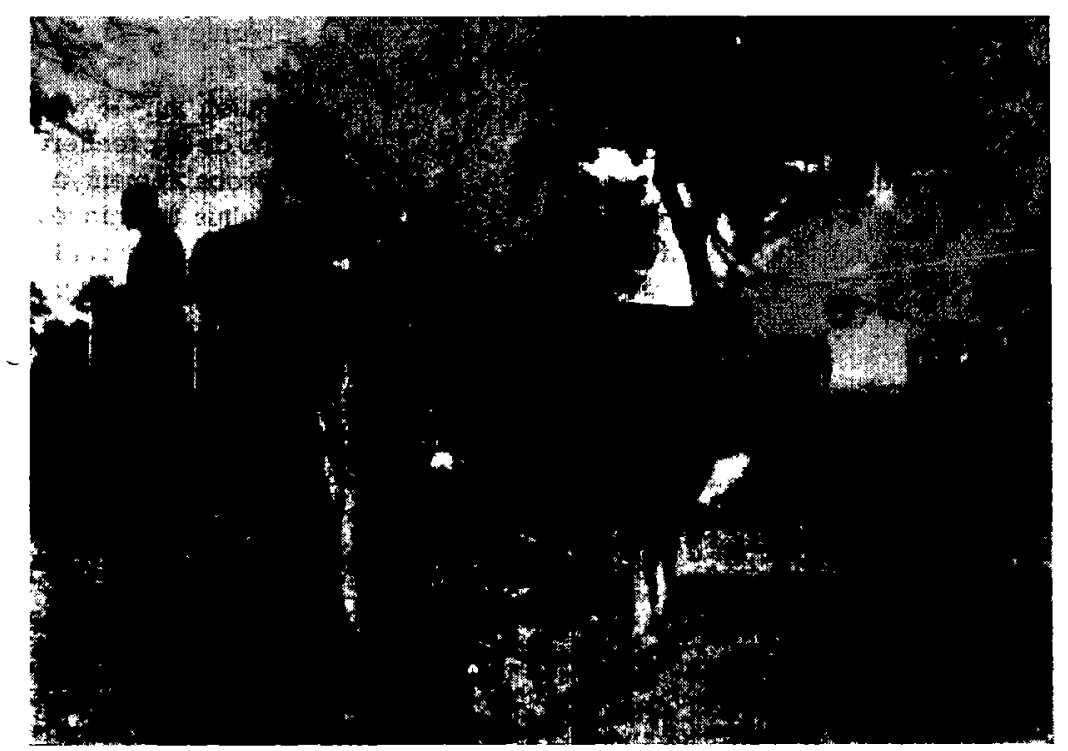

Mère du demi-sang Charollais-n'Dama né le 18,10 2951. 


\section{III. - INSÉMINATIONS ARTIFICIELLES PRATIQUÉES}

I Troupeaux utilisés. Détection des chaleurs. A Bamako, les vaches utilisées étaient celles de la Station d'élevage de Sotuba-Bamako, appartenant à la race N'Dama, itaurins indigènes entretenus à la ferme depuis une quinzaine d'années.

A Ségou, le troupeau de vaches ayant servi aux essais était composé de vaches peules du fleuve, de vaches demi et quart-sang zébu-charollais et zébu-montbéliard.

Trois mois avant le début des inséminations, les vaches, ayant été trouvées presque toutes atteintes de catarrhe granuleux des voies génitales, furent traitées avec une pommade mouillante antiseptique et des injections à base de permanganate. Au début des inséminations, certaines femelles ayant encore des lésions, nous fîmes précéder les inséminations d'une injection bicarbonatée.

Au cours des observations préliminaires, la durée moyenne des cycles observés fut de vingt et un jours (extrêmes 16 et 26 jours) (56 observations).

En A.O.F., les chaleurs de la vache se manifestent en fevrier-mars et de mai à juillet.

A Ségou, nous avons observé que chez les vaches zébu, zébu-charollais, zébu-montbéliard, l'intensité des signes cliniques des chaleurs : écoulement muqueux en particulier, était fonction du degré de sang européen et qu'inversement la régularité des cycles était fonction du degré de sang zébu.

Les signes cliniques étant très discrets chez la plupart des sujets, furent inséminées les vaches présentant des signes cliniques nets de chaleurs " détectées » pár un taureau boute-en-train, ou manifestant au pâturage des signes de désir génésique : recherche du mâle, sauts sur d'autres vaches.

Cependant pour augmenter les chances de succès, cortainos vachos furcnt inséminées, qui n'avaient que des signes très frustes de chaleurs, ou avaient été détectées au pâturage par les bergers.

Techniques d'insémination. - Les inséminations furent faites intracervicales, à l'aide d'un cathéter en verre muni d'une seringue de 2 cc. faisant office de piston. Quelques-unes furent faites intra-utérines. A chaque fois, on utilisa un spéculum, au début un. spéculum à valves, ensuite un spéculum Coldlite en plexiglass. Pour augmenter les chances de succès, les vaches reconnues en chaleur le matin et immédiatement inséminées étaient réinséminées le soir au retour du pâturage; de même, celles inséminées la première fois le soir, l'étaient le lendemain matin.

Résultats. - Nous présentons les résultats obtenus dans les tableaux III et IV.
Comme le montre le tableau III, au cours de 4 séries d'expéditions (2l envois) de sperme de 6 taureaux différents, nous avons inséminé 57 vaches et obtenu 15 fécondations, soit un pourcentage de réussite de $29,1 \%$. Dans la majorité des cas, il a fallu faire au moins 2 inséminations et parfois même de 3 à 5 par vache, soit au total 119 interventions.

On doit noter qu'au cours des 3 premières séries la majorité des inséminations ont été faites à l'aide de sperme récolté depuis plus de quatre-vingtseize heures (tableau IV). Mais le sperme dilué au jaune d'œuf citrate possède, à cet âge, une valeur douteuse pour la fécondation; aussi, afin de calculer les pourcentages de réussite de façon plus juste et plus conforme à la pratique courante de l'insémination artificielle, nous avons rapporté le nombre de vaches fécondées : 15, au nombre des vàches inséminées (au moins pour la première fois) au cours des quatre-vingt-seize heures ayant suivi la rćcolto (38 vaches, voir tableau III). Le pourcentage moyen des fécondations devient alors de $42,3 \%$, très satisfaisant pour le cas d'un transport de semence à longue distance et surtout dans les pays tropicaux.

L'analyse du tableau III suggère aussi d'autres conclusions intéressantes. On voit d'abord que dans la réussite de la fécondation, la saison de l'année joue, en A.O.F., un rôle important.

La période s'étendant du début de décembre jusqu'à la moitié de janvier paraît être la plus favorable $(67 \%$ de vaches fécondées), ainsi que la période d'avril à août ( $56 \%$ et $37,5 \%$ de fécondations). Ces périodes correspondent à peu près al temps de l'apparition des chaleurs chez les vaches indigènes.

Une seule fécondation sur 18 vaches inséminées a été obtenue au cours de la première série d'envois : cet échec est dû probablement à la saison défavorable et aux difficultés techniques, lors du début de nos essais, par exemple, troubles pathologiques des voies 'génitales des vaches indigènes, courte durée des chaleurs, difficulté de leur détection, etc.

Avec un seul envoi comprenant environ $50 \mathrm{cc}$. de sperme dilué, on aurait pu inséminer au moins 30 vaches ou au cours des 21 envois plus de 600 vaches. Cela ne pouvait être réalisé à cause des difficultés locales des communications et du système d'entretien nomade des animaux.

\section{a) Inséminations fécondes (tableau IV).}

Si on calcule l'âge moyen de la semence utilisée lors des inséminations fécondes, en utilisant les premières et secondes inséminations pratiquées sur la même vache à huit ou quatorze heures 'd'intervalle, on trouve qu'il est égal à $67 \pm 5$ heures, et si on n'utilise que les âges de cette semence lors des premières inséminations, on trouve $63 \pm 6$ heures, 


\section{PLANCHE VI}

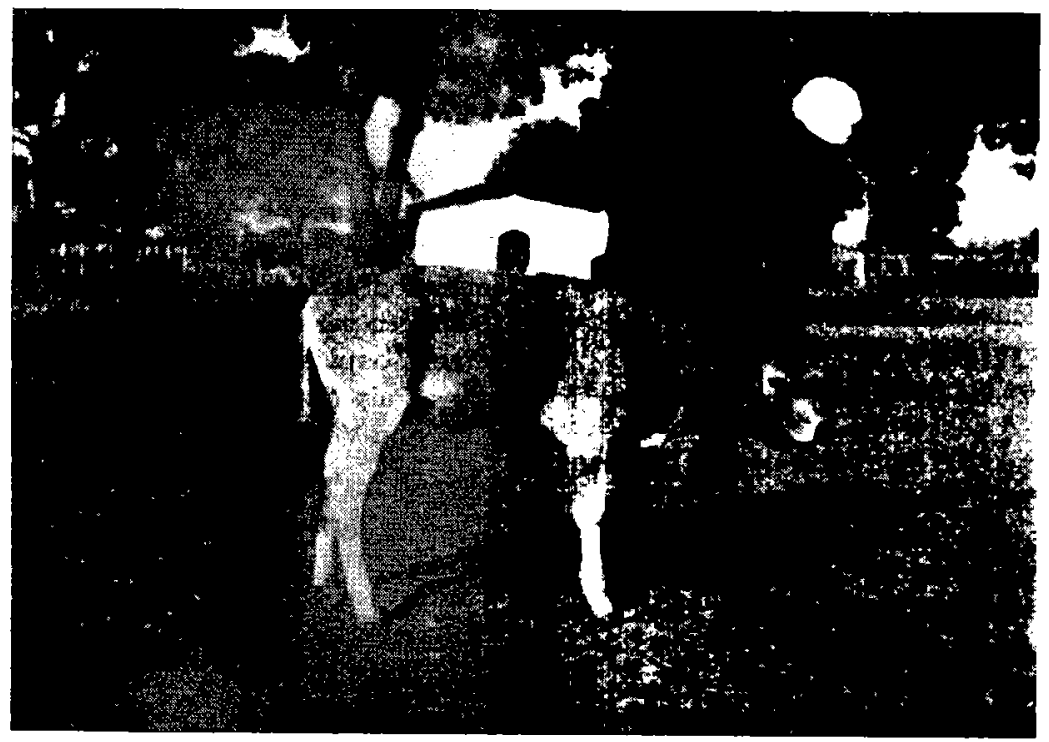

Génisse demi-sang Charollais-n'Dama, née le 18:101951, photographiée à 3 mois.

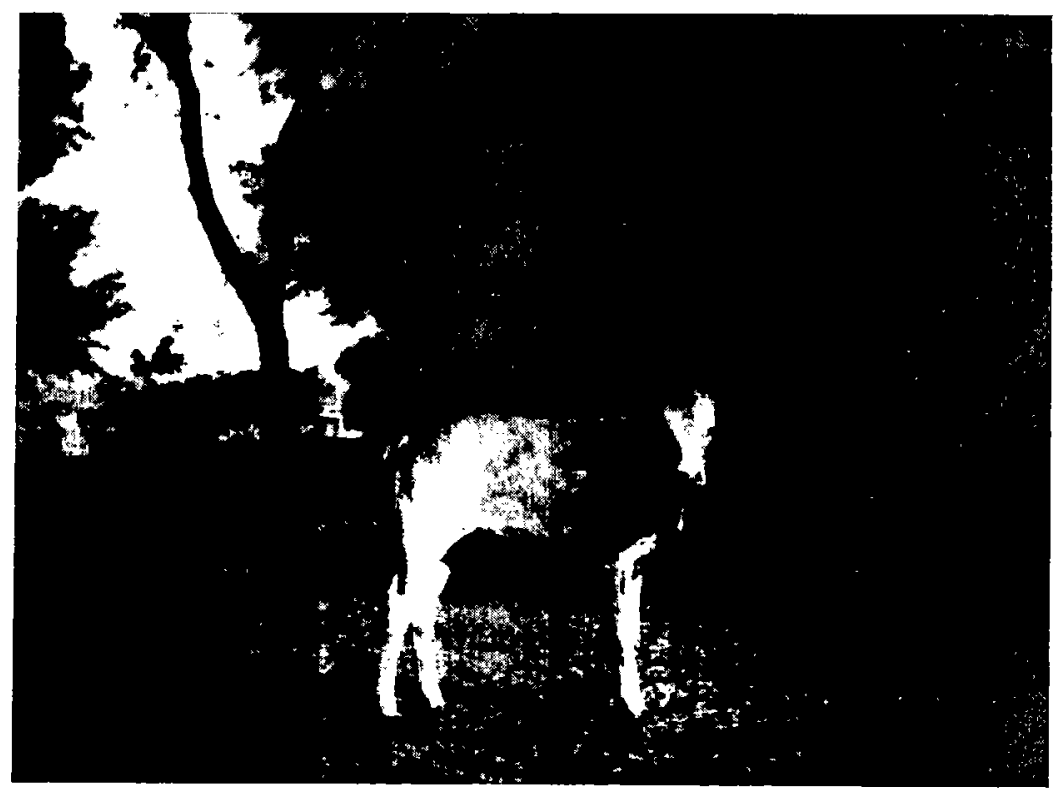

Génisse demi-sang Charollais-n'Dama, née Je 18/10/195I, photographiée à 3 mois. 
moyennes qui ne sont pas significativement différentes. Pour 3 fécondations, les semences étaient âgées de 91,92 et 114 heures au moins et 105 et 124 heures au plus respectivement. Dans un cas, la semence était âgée de 100 heures.

\section{b) Inséminations infécondes (tableau IV).}

Dans la colonne : inséminations infécondes, nous n'avons tenu compte des inséminations pratiquées huit à quatorze heures après la première que lorsque la semence était différente de celle utilisée lors de la première insémination.

On ne peut calculer une moyenne, pour l'âge de la semence; la distribution n'étant pas normale. De plus, on n'a pas eu l'occasion, dans les deuxième et troisieme séries, d'utiliser de la semence âgée de plus d'une semaine (163 heures) et dans la dernière, de plus de 5 jours (107 heures).

La majeure partie des échecs avec des semences âgées de moins de 70 heures l'a été avec des vaches atteintes de troubles pathologiques des voies génitales (dernière série en particulier).

Produits obtenus. - Sur 15 vaches fécondées, 2 sont encore gestantes, 9 ont donné des produits vivants à la naissance, 4 ont avorté. I avortement survenu parmi les vaches inséminécs dans la dornière série fut provoqué par la vaccination contre la peste bovine par un vaccin atténué par passage sur chèvres. Les 3 autres ont une cause indeterminée.

8 des produits métis nés vivants pesaient : demi-schwitz $\times$ N'Dama ........., Mâle, $20 \mathrm{~kg} 500$ demi-tarentais $\times N^{\prime}$ Dama ....... Mâle, 22 kg 500 demi-montbéliard $\times$ N'Dama. . . Femelle, 2.1 kilos

$$
\begin{array}{llll}
- & - & \ldots & \text { Mâle, } 22 \mathrm{~kg} 500 \\
- & - & \ldots & \text { Femelle, } 26 \mathrm{kilos}
\end{array}
$$

demi-charollais $\times$ N̦'Dama...... Femelle, 25 kilos demi-charollais $\times z$ zébu......... Femelle, 22 kilos

On voit que le poids vif des produits métis est au-dessus du poids des veaux nouveau-nés de race N'Dama : $14 \mathrm{~kg} 200$ en moyenne (30 observations).

L'étude du comportement de ces produits au' cours de leur croissance fera l'objet d'un mémoire particulier, quand ils auront atteint l'âge de 3 ans.

\section{IV. - PRIX DE REVIENT ORGANISATION PRATIQUE}

Les résultats de nos expériences ont montré qu'un seul envoi par semaine n'est pas suffisant, mais que deux envois seraient indispensables pour qu'un centre situé outre-mer puisse avoir en permanence de la semence utilisable avec des chances constantes 'de succès.
Le prix unitaire d'un emballage complet s'établit comme suit :

$$
\begin{aligned}
& \text { I bouteille Thermos. } \\
& 2.904 \text { francs } \\
& 1 \text { fût, carton rigide. ........... } \\
& \text { Kapok-coton................. } \\
& \text { Doigtiers caoutchouc (4)....... } \\
& \text { Tubes (4) ................... } \\
& 264 \text { - } \\
& 200- \\
& 200- \\
& 100-
\end{aligned}
$$

Ces emballages sont naturellement récupérables et pourraient être retournés par voie maritime au centre fournisseur moyennant une dépense d'environ 300 francs par emballage et avec un délai d'acheminement depuis Bamako, de trois mois environ.

Les frais d'expédition par avion (exemple pour Magny-en-Vexin) sont les suivants :

Transport Magny-en-Vexin-Paris.

Transit Paris-Orly ou Le Bourget.

Transport avion Paris-Bamako...

Transport Bamako-Sotuba........

250 francs
$500-$
$2.100-$
$100=$
2.950 francs

La coopérative de Magny-en-Vexin ayant accepté de nous céder la semence d'une émission totale au prix exceptionnel de 4.000 francs, chaque envoi reçu à Bamako revenait à 10.618 francs métropolitains, soit 5.309 francs C.F.A.

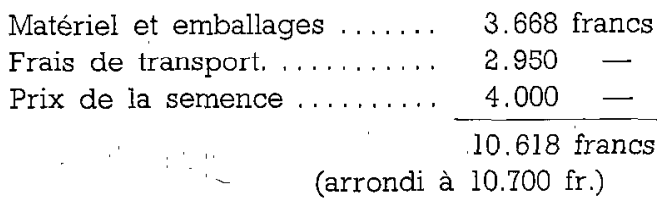

Si le nombre de vaches destinées à l'insémination artificielle est suffisant pour qu'à chaque envoi on puisse espérer quelques fécondations, l'opération est parfaitement rentable. A cela s'ajoute la valeur des renseignements fournis par l'étude du comportement des produits issus du métissage et qui peuvent contribuer à dégager des notions intéressantes pour l'élevage sous les tropiques.

\section{CONCLUSIONS}

Notre but était de déterminer les possibilités pratiques d'emploi de l'inséminlation de vaches indigènes avec de la semence expédiée de France.

21 envois de semences de . France sur Bamako ont permis de pratiquer, depuis 1949, 119 inséminations sur 57 vaches dont 15 ont été fécondées.

Le pourcentage de fécondation pour toutes les vaches inséminées est de $29,7 \%$; pour les vaches inséminées avec du sperme âgé đe moins de 96 heures, il atteint $42,3 \%$. Les résultats de ces expériences initiales, pratiquées sur des vaches 


\section{PLANCHE VII}

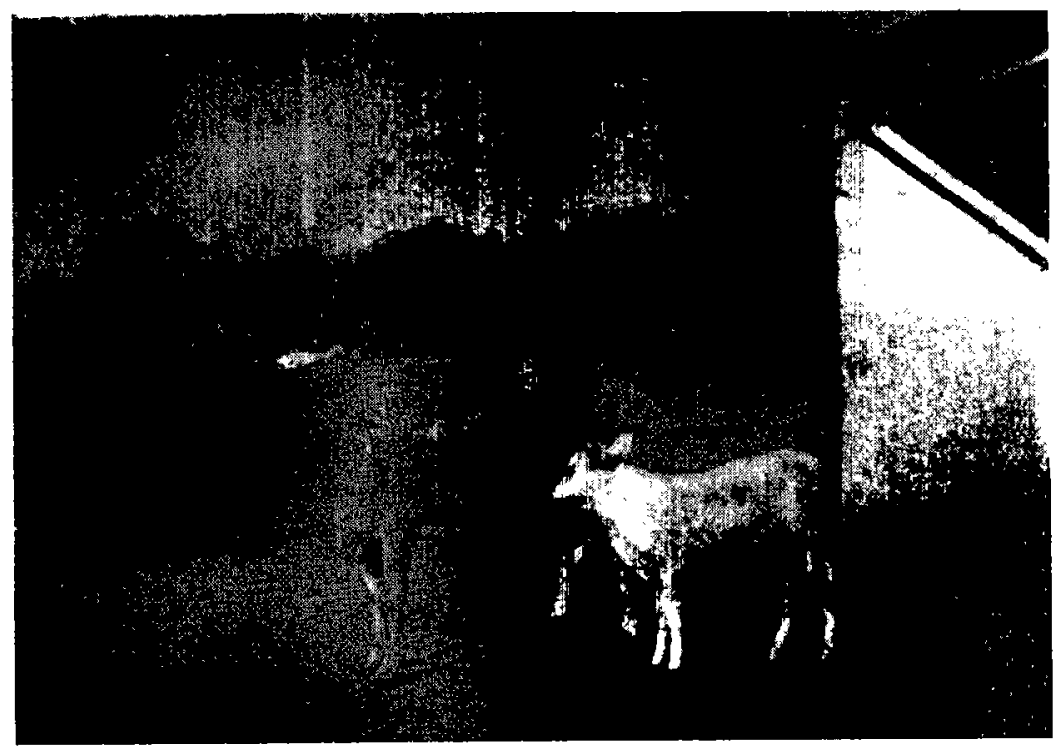

Vache demi-sang Charollais-Zébu et son produit charollais 3/4 né le 2/9/1951 (Génisse ágée de 4 mois).

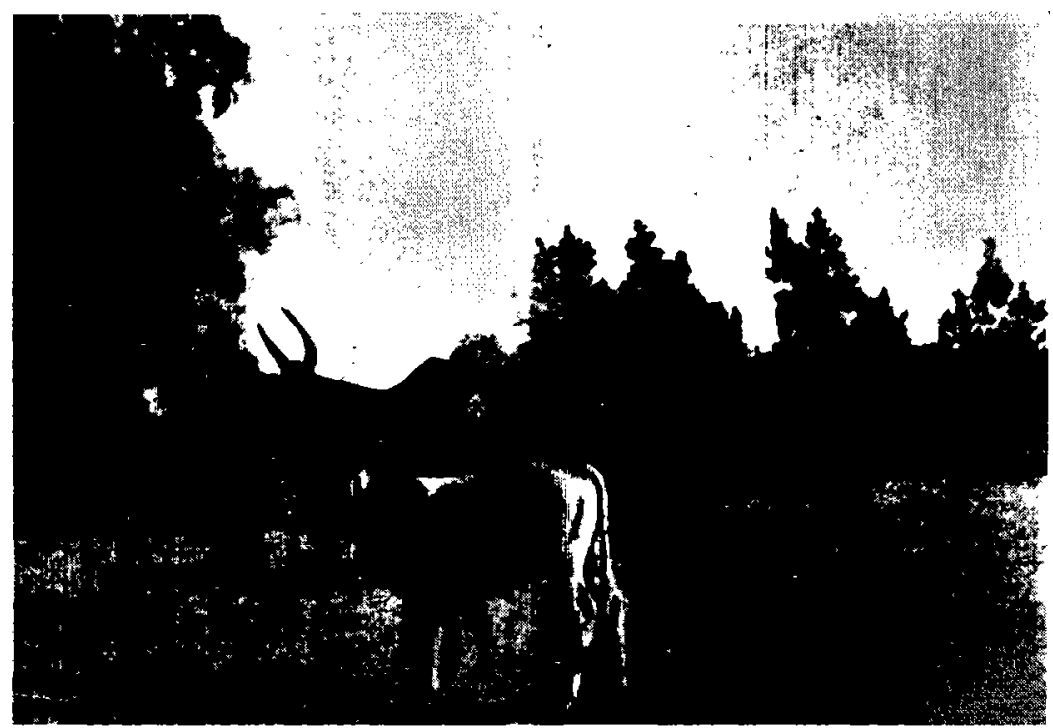

Vache zşbu et son produit demi-sang, né le 14/10;1950 (Génisse ágée de 3 mois). 


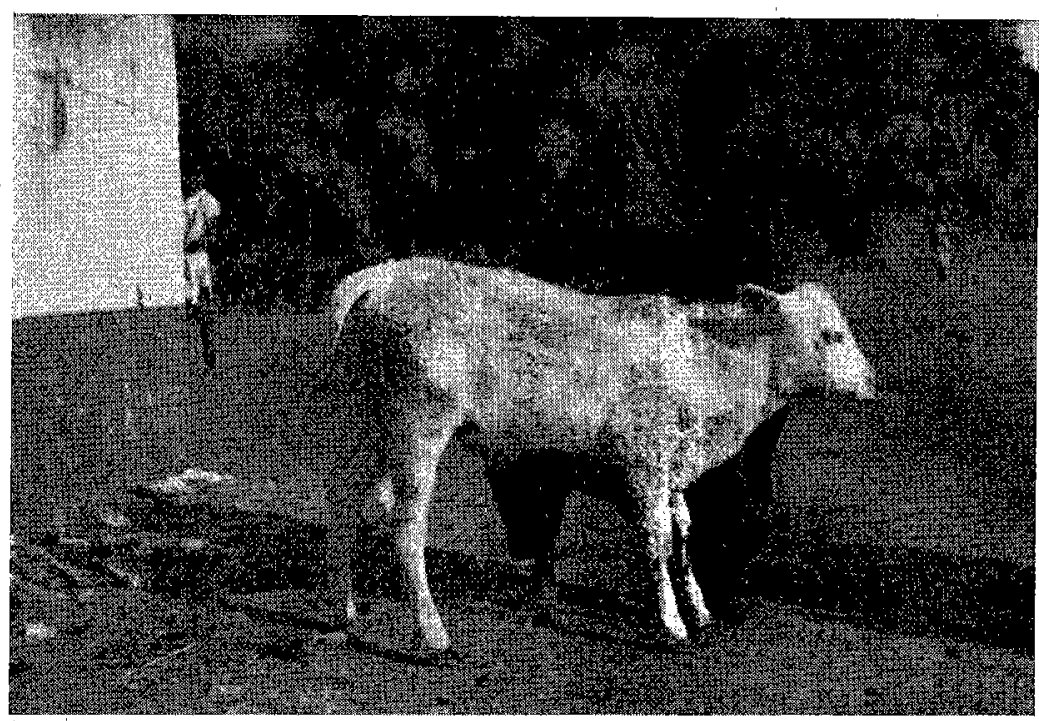

Génisse trois-quarts-sang Charollais-zébu, née le 2/9/1951, photographiée à l'âge de 4 mois.

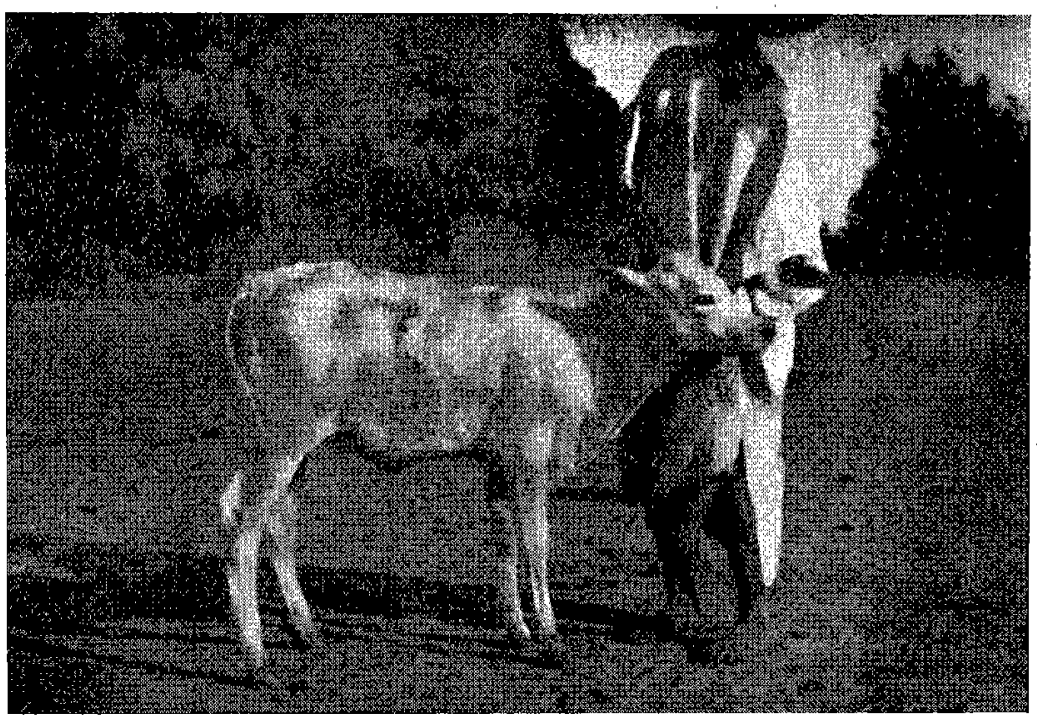

Génisse demi-sang Charollajs-zébu, née le 14/10/1951, photographiée à l'âge de 3 mois. 


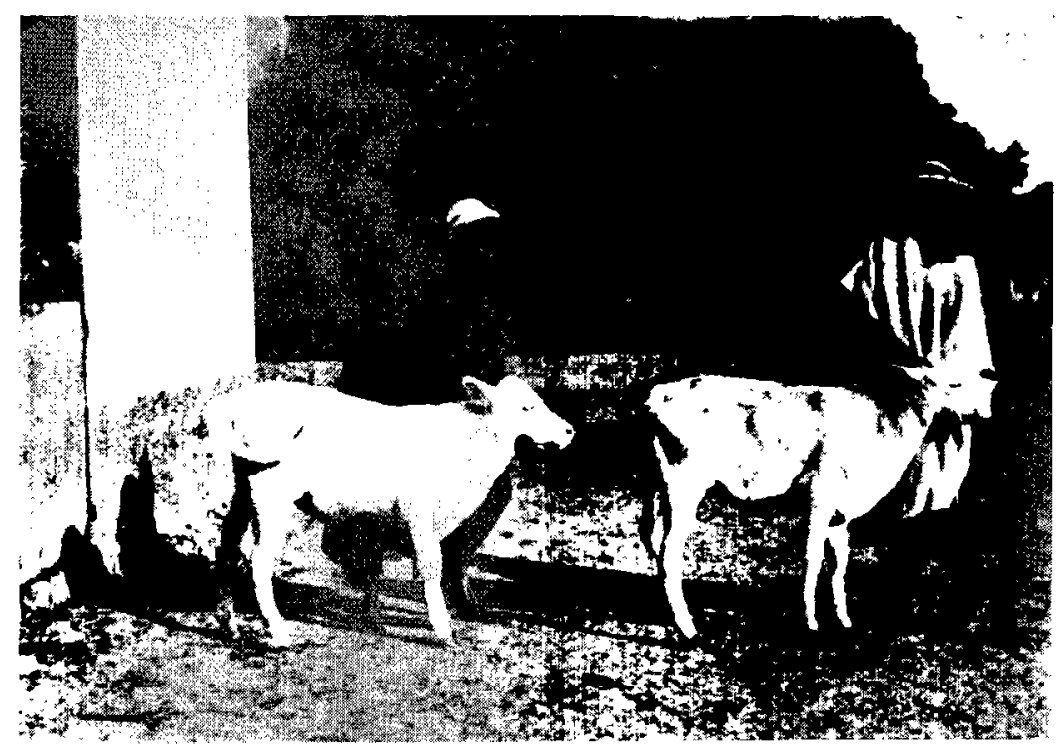

Génisses trois-quart et demi-sang Charollais-zébu, photographiées respectivement à 4 et 3 mois à Ségou.

qui n'étaient pas toujours dans les conditions les plus favorables à la réussite, peuvent être considérés comme tout à fait satisfaisants.

Nous pensons avoir confirme "efficacite des méthodes de conditionnement e: c'expécition dont le détail a été antérieurement pliolie (1). Peut-être pourra-ton trouver encore quelques iggers perfectionnements, mais ils ne modifieroni certainement pas beaucoup la technique exposée ci-dessus.

Un centre situé à moins de 200 kilonètres d'un aérodrome desservi régulieremen: 'Bamako, Niamey, Dakar, Bouaké, Abidjanj peut ètre facilement ravitaille par un centre de la région parisienne et avoir en permanence de la semence âgée de moins de 96 heures, ce qui, avec les novveaux dilueurs (lait écrémé), est parfaitement suffisant pour son emploi pratique.

C'est pour les inséminations elles-mêmes que les difficultés sont les plus grandes. car les chaleurs de vaches indigènes sont irrégulières, courtes et

(1) LETARD, SZUMOWSKI et FRRUTI. - Conaitionnement pour le transport du sperme à longue distance. C. R. Acad. Agric. novembre 1949 . discrètes. Il conviendrait que les envois de sperme fussent zius frequents (bihebdomadaires) et exécutés aux saisons ou se manifestent d'ordinaire les chaleurs des ímeiles en A.O.F. (janvier-fóvrier of avril-août).

I. Sotuca comme à Ségou, nous sommes arrivés aux mêmes conclusions; c'est dans la technique d'entretien des animaux qu'est maintenant le problème le plus important à résoudre.

Dans ces deux stations, l'élevage semi-extensif en paturage ouvert ne permettait pas l'observation permanente des animaux par du personnel qualifié. On re pouvait faire les examens que le matin au depart, et le soir au retour du pâturage; certaines vaches ont été inséminées parce que "vues 》 en chaleurs au pâturage par les bergers peuls. Avec les pâturages ouverts, on doit redouter le danger de saillies par des taureaux étrangers au Centre Expérimental.

Pour avoir de nombreuses inséminations fécondes, il faut d'abord avoir un troupeau très important de vaches vides, au moins 200 et il faut qu'elles puissent être observées en permanence pour que soient reconnus les premiers signes d'apparition des chaleurs. 
Chez les femelles où les manifestations sexuelles sont très peu apparentes, on pourrait tenter un traitement hormonal.

Pour ce but, il faut que le Centre utilisateur possède un petit pâturage clos de bonne qualité dans. lequel on pourra mettre les vaches à examiner avec un taureau vasectomisé, métis taurin européen de préférence et on les observera tout au long de la journée. Le maintien à l'étable n'est pas recommandé.
L'époque des « expériences » est à notre avis terminée. Si les conditions d'emploi que nous précisons ci-dessus sont remplies, l'utilisation pratique de l'insémination artificielle avec de la semence importée de France en A.O.F. pour I'obtention de métis est possible.

Nous tenons à adresser ici nos vifs remerciements à la Compagnie Air France, qui a facilité nos essais avec la plus grande amabilité et a assuré le transport du sperme avec. une parfaite diligence. 